\title{
BMJ Open Assessing the effectiveness of perioperative s-ketamine on new-onset headache after resective epilepsy surgery (ESPAIN-trial): protocol for a randomised, double-blind, placebo- controlled trial
}

Jiske Cornelia Theresa Sloekers, ${ }^{\oplus 1}$ Michael Bos, ${ }^{2}$ Govert Hoogland, ${ }^{1,3}$ Caroline Bastiaenen, ${ }^{4}$ Sander van Kuijk, ${ }^{5}$ Maurice Theunissen, ${ }^{2}$ Kim Rijkers, ${ }^{1}$ Jim Dings, ${ }^{1}$ Albert Colon, ${ }^{6,7}$ Rob P W Rouhl, ${ }^{8}$ Olaf Elisabeth Maria Ghislaine Schijns ${ }^{1,3}$
To cite: Sloekers JCT, Bos M, Hoogland $\mathrm{G}$, et al. Assessing the effectiveness of perioperative s-ketamine on new-onset headache after resective epilepsy surgery (ESPAIN-trial): protocol for a randomised, double-blind, placebocontrolled trial. BMJ Open 2019;9:e030580. doi:10.1136/ bmjopen-2019-030580

\section{- Prepublication history for} this paper is available online. To view these files please visit the journal online (http://dx.doi org/10.1136/bmjopen-2019030580).

Received 25 March 2019 Revised 23 July 2019 Accepted 29 July 2019
Check for updates

(C) Author(s) (or their employer(s)) 2019. Re-use permitted under CC BY-NC. No commercial re-use. See rights and permissions. Published by BMJ.

For numbered affiliations see end of article.

Correspondence to Jiske Cornelia Theresa Sloekers; jiske.sloekers@mumc.nl

\section{ABSTRACT}

Introduction Effective treatment of new-onset headache after craniotomy, especially anterior temporal lobectomy (ATL) and amygdalohippocampectomy for drug-resistant temporal lobe epilepsy, is a challenge. The current practice, acetaminophen combined with opioids is often reported by patients as insufficient and sometimes accompanied by opioid-related adverse effects. Based on expert opinion, anaesthesiologists therefore frequently consider s-ketamine as add-on therapy. This randomised parallel group design trial compares s-ketamine with a placebo as add on medication to a multimodal pain approach.

Methods and analysis In total 62 adult participants, undergoing ATL for drug resistant epilepsy under general anaesthesia, will be randomised to either receive a $0.25 \mathrm{mg} / \mathrm{kg}$ bolus followed by a continuous infusion of $0.1 \mathrm{mg} / \mathrm{kg} /$ hour of s-ketamine or placebo $(0.9 \% \mathrm{NaCl})$ starting before incision and continued for 48 hours as an addition to acetaminophen and opioids administered in a patient-controlled analgesia pump. The primary outcome measure is the cumulative postoperative opioid consumption. Patient recruitment started August 2018 and will end in 2021. Secondary outcome measures are postoperative pain intensity scores, psychological parameters, length of hospital stay and adverse events and will be reassessed at 3 and 6 months after surgery, with a baseline measurement preoperatively. All data are collected by researchers who are blinded to the treatment. The data will be analysed by multivariable linear mixedeffects regression.

Ethics and dissemination Ethical approval has been given by the local medical ethical committee (NL61666.068.17). This study will be conducted in accordance with the Dutch Medical Research Involving Human Subjects Act and the Declaration of Helsinki. The results of this trial will be publicly disclosed and submitted for publication in an international peer-reviewed scientific journal.
Strengths and limitations of this study

- First randomised controlled trial to evaluate the effect of s-ketamine as add-on analgesic therapy in treating new-onset headache after craniotomy in a strict uniform study group: patients with temporal lobe epilepsy undergoing anterior temporal lobectomy under general anaesthesia.

- The surgical technique and the anaesthesiologic procedure are the same in all patients and performed by the same surgeons (OS and JD) and a small group of neuroanaesthesiologists.

- Extensive measurement at baseline and follow-up of relevant factors that influence pain perception enables more precise interpretation of pain intensity scores.

- The finding of a positive effect of s-ketamine as add-on analgesic therapy will contribute to the conceptualisation of a standardised guideline for the treatment of postcraniotomy headache.

- A limitation is that opioid consumption, though an objective and quantifiable measure, does not necessarily reflect a clinically relevant effect in the treatment of postcraniotomy headache.

Trial registration number NTR6480.

\section{INTRODUCTION}

Until recently, clinicians strongly believed that patients had minimal pain and discomfort following craniotomy, based on low average pain intensity scores in a frequently cited retrospective study. However, their results were based only on a short $(90 \mathrm{~min})$ postoperative observation of patients who received substantial intraoperative 
analgesics. ${ }^{1}$ Contrarily, postcraniotomy headache is the most common adverse event following craniotomy. The reported incidence is highly variable, rising up to $91 \%$ depending on the criteria used for the definition of postcraniotomy headache. ${ }^{2-5}$ According to the International Classification of Headache Disorders (third edition) by the International Headache Society, postcraniotomy headache is defined as a headache that develops within 7 days after craniotomy. Acute postcraniotomy headache resolves within 3 months, whereas the chronic form persists for $>3$ months. ${ }^{6}$ According to this definition, the reported incidence of postcraniotomy headache is $40 \%$, whereby $10.7 \%$ was limited to the acute stage and $29.3 \%$ developed into a chronic form. ${ }^{7}$ When the definition is broadened to onset within 30 days, the reported incidence rises to $62 \%$ and when including headache anytime during follow-up, the incidence reported is $91 \% .^{3}$ The incidence of postcraniotomy headache after temporal lobectomy for focal drug-resistant epilepsy is reported as $17.5 \%$ for headaches that persist for $>2$ months and $11.9 \%$ for $>1$ year. ${ }^{8}$ This is unfavourable as chronic headaches are often associated with symptoms of depression and anxiety that in turn interfere with quality of life. ${ }^{79}$

Since the pathophysiology of postcraniotomy headache is not well understood, it is generally poorly managed. ${ }^{2}{ }^{10}$ Acetaminophen is often not sufficient and the use of non-steroidal anti-inflammatory drugs in neurosurgical procedures is controversial due to their effect on bleeding time. ${ }^{11}$ The current practice is opioids combined with acetaminophen, yet there still is continuing controversy regarding the choice of the 'best' regimen. ${ }^{101213}$ The use of potent analgesic opioid drugs, administered by a patient-controlled analgesia (PCA) pump, may cause serious side effects such as respiratory depression, vomiting, nausea, ileus and may lead to deterioration of consciousness, which interferes with the assessment of the neurological status and postcraniotomy pain. ${ }^{10}$

The addition of other non-opioid analgesics, such as s-ketamine, might be effective in reducing the need for opioid analgesics. The analgesic effects of s-ketamine are mediated by an N-methyl-D-aspartate-receptor antagonist mechanism, which improves the efficacy of opioids and reduces the development of chronic pain syndromes. ${ }^{14-17}$ When used in high doses, this drug is known for its reversible neuropsychiatric side effects such as hallucinations, nightmares and blurred vision. However, when s-ketamine is administered in low doses, these side effects are well tolerated, reversible and occur in a minority of patients. ${ }^{14}$ Benefits of s-ketamine in postoperative pain management and its opioid sparing effects have already been reported in abdominal, thoracic and orthopaedic surgeries. ${ }^{1418}$ The most recent systematic review on the use of s-ketamine in craniotomies showed an overall opioid sparing effect of $40 \%$. However, the effect on pain intensity scores was very diverse, whereby only 8 of 34 studies showed a significant reduction. The majority of the studies used was underpowered for pain intensity scores, the assessment of pain intensity scores differed largely and factors that influence pain perception, such as anxiety and depression, were poorly documented. ${ }^{19}$

To date, no studies have been performed to evaluate the beneficial effects of s-ketamine as part of a multimodal pain approach after anterior temporal lobectomy (ATL) for drug-resistant temporal lobe epilepsy (TLE). The primary objective of this randomised parallel group trial is to investigate the effect of perioperative s-ketamine, compared with placebo, as addition to a routine multimodal pain approach of acetaminophen and opioids, on the total opioid consumption after craniotomy in patients with drug-resistant TLE. In this paper, we present the study protocol according to Standard Protocol Items: Recommendations for Interventional Trials protocol guidelines for randomised controlled trial protocols.

\section{PATIENTS AND METHODS}

\section{Design and setting}

The ESPAIN trial is a randomised, placebo-controlled, double-blind, single-centre trial with two parallel groups. Patients with drug-resistant TLE scheduled for elective ATL with or without amygdalohippocampectomy under general anaesthesia are suitable candidates for study participation. The surgical technique is the same in all included patients and the surgery is performed by either of two neurosurgeons (OS and JD) from the department of Neurosurgery of the Maastricht University Medical Centre+ (MUMC+), Maastricht, the Netherlands.

\section{Recruitment, eligibility criteria and informed consent}

Approximately 21 TLE patients are scheduled for surgery every year in the neurosurgical outpatient clinic of the MUMC+. All patients scheduled for elective ATL with or without amygdalohippocampectomy will be considered for eligibility. Prior to their visit to the outpatient clinic, these patients will be informed about their upcoming operation by one of the three epileptologists at Kempenhaeghe Academic Centre for Epileptology, Heeze, the Netherlands. The epileptologist will ask patient's permission for a phone interview by the GCP-certified coordinating investigator (JS). In this phone call, the patient will receive detailed study information and will be asked for permission to send a patient information brochure by mail. The patient will have a minimum of 14 days to consider participation, starting from the moment of receiving the patient information brochure at home. During the visit at the neurosurgical outpatient clinic of the MUMC+, the patient will be informed about the surgery by one of the two neurosurgeons who perform the epilepsy surgery. Afterwards, the coordinating investigator (JS) will answer any remaining questions about the trial. When it is clear that the patient has understood all the provided information and wants to participate in the trial, both the patient and the coordinating investigator will sign the informed consent sheet, after which the patient will be included in the randomisation procedure. 
Patients eligible for the trial must comply with all of the inclusion criteria and are not allowed to have any of the exclusion criteria, as listed in table 1 . The general practitioner will be informed by letter about the patient's participation in the ESPAIN trial.

Resective temporal lobe surgery is the golden standard curative therapy for drug-resistant TLE. Novel methods such as MRI guided laser institutional thermal therapy are not yet available in the Netherlands, only stereoelectroencephalography-guided radiofrequency thermocoagulation is applied for highly selected patients. Therefore, we do not expect a drop in participant recruitment.

\section{Interventions}

Patients will be randomly allocated to either of the two study arms. The intervention group receives s-ketamine (Ketanest-S, Pfizer, Dun Laoghaire, Ireland) and the control group receives a placebo $(0.9 \% \mathrm{NaCl})$. The operation consists of an ATL with or without an amygdalohippocampectomy performed with a standard surgical technique for all patients by either of two neurosurgeons (OS and JD). The trial medication will start perioperatively after induction, but before incision, and will be continued for 48 hours in total. The intervention group will receive a bolus of $0.25 \mathrm{mg} / \mathrm{kg}$ s-ketamine, followed by a continuous infusion of $0.1 \mathrm{mg} / \mathrm{kg}$. This dosage is based on the Dutch Association of Anaesthesiology guidelines on postoperative pain The placebo $(\mathrm{NaCl} 0.9 \%)$ will be administered in the same dosage and packaging as s-ketamine. All patients will receive general anaesthesia as part of the routine medical treatment as further specified in the overview of interventions in figure 1. No

\begin{tabular}{|c|c|}
\hline Inclusion criteria & Exclusion criteria \\
\hline Age $>18$ years & Declined informed consent \\
\hline $\begin{array}{l}\text { Elective resective surgery for } \\
\text { drug-resistant temporal lobe } \\
\text { epilepsy }\end{array}$ & $\begin{array}{l}\text { Allergy to any of the trial } \\
\text { medications }\end{array}$ \\
\hline \multirow{3}{*}{$\begin{array}{l}\text { Drug-resistant epilepsy, } \\
\text { based on: } \\
\text { Chronic, focal epilepsy. } \\
\text { Not seizure free with } \\
\text { antiepileptic medication. } \\
\text { No medication options } \\
\text { due to adverse effects. }\end{array}$} & $\begin{array}{l}\text { Current chronic pain, such as, } \\
\text { but not limiting to, migraine or } \\
\text { other headaches }\end{array}$ \\
\hline & $\begin{array}{l}\text { Chronic pain treatment with } \\
\text { use of different kinds of pain } \\
\text { medication }\end{array}$ \\
\hline & $\begin{array}{l}\text { Alcohol, hard or soft drug } \\
\text { abuses }\end{array}$ \\
\hline \multirow[t]{3}{*}{$\begin{array}{l}\text { Signed informed consent for } \\
\text { trial participation }\end{array}$} & $\begin{array}{l}\text { Inability to complete } \\
\text { questionnaires or language } \\
\text { barrier }\end{array}$ \\
\hline & $\begin{array}{l}\text { History of psychiatric } \\
\text { complaints for which } \\
\text { treatment was performed }\end{array}$ \\
\hline & $\begin{array}{l}\text { History of craniotomy } \\
\text { or subdural electrode } \\
\text { implantation }\end{array}$ \\
\hline
\end{tabular}

premedication will be given, except for the patient's own antiepileptic medication.

In case the potential side effects of s-ketamine cause too much discomfort for patients, this will be registered as an adverse event. In case of severe hallucinations, the study medication will be terminated and the PCA pump and acetaminophen will be continued. The patient will not be deblinded or withdrawn from the study. Data collection will continue according to the protocol.

In case a patient experiences severe pain, the study medication will be discontinued and regular s-ketamine protocol will be started. This consists of morphine in a PCA pump, acetaminophen and s-ketamine. Since the patient already receives morphine in a PCA pump and acetaminophen, only the study medication will be discontinued and replaced with s-ketamine. S-ketamine is chosen as rescue medication, since it is a potent analgesic

\begin{tabular}{|c|c|}
\hline \multicolumn{2}{|c|}{$\begin{array}{l}\text { Application of standard monitoring } \\
\text { First } 18 \mathrm{G} \text { intravenous line to start crystalloid infusion } \\
\text { Second } 18 \mathrm{G} \text { intravenous line for anaesthetics }\end{array}$} \\
\hline \multicolumn{2}{|c|}{$\nabla$} \\
\hline \multicolumn{2}{|c|}{$\begin{array}{l}\text { Induction: } \\
\begin{aligned} \text { - } & \text { Bolus sufentanil } 0.2-0.4 \mu \mathrm{g} / \mathrm{kg} \text { intravenous } \\
\text { - } & \text { Propofol } 2 \mathrm{mg} / \mathrm{kg} \text { intravenous } \\
\text { - } & \text { Rocuronium } 0.6 \mathrm{mg} / \mathrm{kg} \text { intravenous } \\
\text { - } & \text { Dexamethasone } 10 \mathrm{mg} \text { intravenous }\end{aligned}\end{array}$} \\
\hline \multicolumn{2}{|c|}{$\nabla$} \\
\hline \multicolumn{2}{|c|}{$\begin{array}{l}\text { Oral intubation and ventilation: } \\
-\quad \text { Male tube size } 8.0 \text {, female tube size } 7.5 \\
\text { - } \\
\text { Tidal volumes } 6-8 \mathrm{~mL} / \mathrm{kg} \text {, frequency } 12-15 \\
\text { per minute, } 5 \mathrm{~cm} \mathrm{H} \mathrm{H}_{2} \mathrm{O} \text { positive end- } \\
\text { expiratorv pressure [PEEPI) }\end{array}$} \\
\hline \multicolumn{2}{|c|}{$\nabla$} \\
\hline \multicolumn{2}{|c|}{$\begin{array}{l}\text { Maintenance: } \\
\begin{aligned} \text { - } & \text { Propofol } 5-7 \mathrm{mg} / \mathrm{kg} / \text { hour intravenous } \\
\text { - } & \text { Remifentanil } 0.25-0.5 \mu \mathrm{g} / \mathrm{kg} / \mathrm{min} \text { intravenous }\end{aligned}\end{array}$} \\
\hline \multicolumn{2}{|c|}{$\begin{array}{c}\text { Blood pressure: in range of } 20 \% \text { from baseline: } \\
-\quad \text { Hypotension: noradrenaline } 0.05-0.1 \\
\mu \mathrm{g} / \mathrm{kg} / \mathrm{min} \\
-\quad \text { Hypertension: nicardipine } 3-5 \mathrm{mg} / \text { hour, } \\
\text { maximum } 15 \mathrm{mg} / \text { hour }\end{array}$} \\
\hline \multicolumn{2}{|c|}{$\downarrow$} \\
\hline \multicolumn{2}{|c|}{$\begin{array}{l}\text { Trial medication: after induction, before incision. } \\
\text { Continued } 48 \text { hours postoperatively. }\end{array}$} \\
\hline $\begin{array}{l}\text { Bolus s-ketamine } 0.25 \mathrm{mg} / \mathrm{kg} \text {, } \\
\text { with continuous s-ketamine } \\
\text { infusion of } 0.1 \mathrm{mg} / \mathrm{kg} / \text { hour. }\end{array}$ & $\begin{array}{l}\text { Bolus } 0.9 \% \mathrm{NaCl} \text {, with } \\
\text { continuous } 0.9 \% \mathrm{NaCl} \\
\text { infusion, both in the same } \\
\text { packaging and volume as s- } \\
\text { ketamine }\end{array}$ \\
\hline \multicolumn{2}{|c|}{$\begin{array}{c}30 \text { minutes before end of surgery: loading dose } \\
\text { morphine } 0.1 \mathrm{mg} / \mathrm{kg} \text { intravenous }\end{array}$} \\
\hline \multicolumn{2}{|c|}{$\downarrow$} \\
\hline \multicolumn{2}{|c|}{ 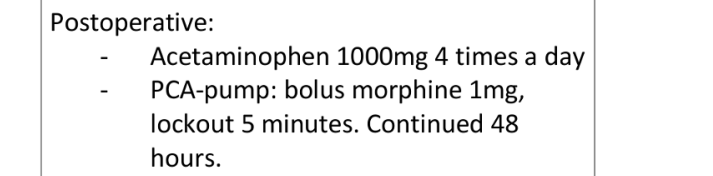 } \\
\hline
\end{tabular}


drug and the current practice for postoperative rescue pain management is already based on use of s-ketamine. Increasing the morphine dosage in the PCA pump is undesirable due to the subsequent increase in adverse events caused by opioids. Therefore, we have chosen to apply the current practice of s-ketamine as rescue medication. The patient will not be deblinded and data collection will continue according to protocol. Severe pain will be defined as unsatisfactory pain treatment despite receiving the maximum amount of morphine with the highest possible PCA pump dosage for at least 1 hour. When the trial medication is discontinued and regular s-ketamine protocol is started, the time, pain score (Numeric Rating Scale (NRS) and Visual Analogue Scale (VAS)) and the reason for discontinuation of trial medication will be registered.

\section{Outcomes}

The primary study outcome is the total postoperative opioid consumption (mg) in both study arms, measured at the seventh postoperative day, with interim measurements at 24, 48, 72 and 96 hours postoperatively.

Secondary study outcomes are length of hospital stay (days), postoperative pain intensity scores from day 0 until 4 and at day 7 postoperatively, and the occurrence of several potential adverse events. Pain intensity is quantified in scores using the VAS, range $0-100 \mathrm{~mm}$, and NRS, range $0-10$. In case pain intensity scores and the accompanying opioid consumption achieve paradoxical results, implications of the effect of s-ketamine on postoperative headache will be based on the opioid consumption, since it is the main outcome measure. Specifically, the occurrence of delirium as adverse event is assessed every day for the first seven postoperative days using the delirium observation screening (DOS) scale. Other potential adverse events that will be registered are hallucinations, nausea, vomiting, dizziness, vivid dreams or nightmares, diplopia, blurred vision, nystagmus and elevated blood pressure or heart rate. Furthermore, patient health-related quality of life, surgical fear, depression, pain catastrophising, severity of pain, neuropathic pain and characteristics of the headache will be assessed using standardised and validated questionnaires as presented in table 2.

The reliability and validity of the NRS and VAS scoring systems is high and both scores are recommended as sensitive and responsive in measuring pain intensity. ${ }^{20}$ The DN4-interview is a diagnostic tool that enables physicians to make a distinction between nociceptive and neuropathic pain and is linguistically validated for usage in Dutch, ${ }^{21}$ whereby only the seven self-report items will be used (DN4-interview) ${ }^{22}$ The CES-D is a screening tool for depression with excellent validity. ${ }^{23}$ Furthermore, the SFQ, PCS, RAND-36, BPI-SF and DOS have also been proven valid and reliable. ${ }^{24-28}$ The self-compiled questionnaire inquiring the characteristics of the headache was compiled by a headache neurologist, based on the International Classification of Headache Disorders-third edition by the International Headache Society.

Other study parameters which will be documented are baseline values such as gender, age, weight, type of antiepileptic drugs, epilepsy duration, details of the surgery, duration of surgery, side of surgery and seizure outcome according to International League Against Epilepsy and Engel class.

\section{Participant timeline}

The study duration for each single patient is 6 months, starting at the moment of trial inclusion until the last questionnaire has been completed 6 months later, as can be seen in figure 2 . The baseline questionnaires will be provided web-based to the surgical candidates before hospital admission and will take $\sim 45 \mathrm{~min}$ to complete.

Table 2 Abbreviations and timing of questionnaires

\begin{tabular}{|c|c|c|c|}
\hline RAND-36 & \multicolumn{3}{|c|}{ Research and development-36-item health survey } \\
\hline SFQ & \multicolumn{3}{|c|}{ Surgical Fear Questionnaire } \\
\hline CES-D & \multicolumn{3}{|c|}{ Centre for Epidemiologic Studies Depression scale } \\
\hline BPI-SF & \multicolumn{3}{|c|}{ Brief Pain Inventory-Short Form } \\
\hline DN4-interview & \multicolumn{3}{|c|}{ Douleur Neuropathique (seven self-report items) } \\
\hline RAND-36 & $\mathrm{X}$ & $x$ & 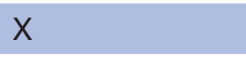 \\
\hline SFQ & $\mathrm{X}$ & & \\
\hline CES-D & $\mathrm{X}$ & $x$ & $\mathrm{X}$ \\
\hline PCS & $x$ & & \\
\hline
\end{tabular}


After surgery, at day 1 until 4 and at day 7 , patients will complete a pain diary and anaesthesiologic nurse practitioners and nurses on the neurosurgical ward will report VAS and NRS scores and presence of delirium using the DOS questionnaire. Completing the pain diary will take $\sim 15$ min each day. The last two follow-up questionnaires will be completed web-based after 3 and 6 months. The total burden of study participation for the patient will be 3 hours.

\section{Sample size}

A minimum of 62 patients is needed for the study sample, with 31 patients in each study arm. A 5\% dropout rate has been included in the calculation. The sample size calculation is based on clinical data from previous patients who underwent resective epilepsy surgery for TLE and were administered s-ketamine as pain medication. It is performed with Gpower based on longitudinal analysis of the data with five measurements, and based on the primary outcome: a difference of standards of opioid consumption. Expected means and SD for each group (intervention group $32 \mathrm{mg}$ and control group $42 \mathrm{mg}$ of opioid consumption (SD $24 \mathrm{mg}$ )), with an effect size based on Cohen d of 0.29, lead to a sample size of $n=58$. Presuming a level of significance $<5 \%$ and a power of 0.80 , a minimum of 58 patients is needed, with the addition of 4 people for an expected drop out of $5 \%, 62$ people have to be included in the trial. With an expectation of 21 eligible trial participants every year, the duration of the period of inclusion will be 3 years.

\section{Randomisation, allocation and blinding}

Allocation of study participants will be done by randomisation by use of a computerised randomisation system at the Department of Epidemiology, Maastricht University. Block randomisation with random permuted block sizes of 4 and 6 will be used. The randomisation scheme will be handed over by an independent member of the Department of Epidemiology to the hospital pharmacist. The surgeon, the attending anaesthesiologist, the nurse, the patient and all investigators will be blinded to the allocated study treatment. The hospital pharmacist, the single not-blinded trial staff member, will prepare the medication according to the allocated protocol either for s-ketamine or $\mathrm{NaCl} 0.9 \%$ in the correct dosage for the correct patient and will ensure that the study medication is labelled and can be administered by nursing staff in a blind manner. In case emergency unblinding is required, the investigator will contact the hospital pharmacist, who is the only trial staff member authorised to trace back to which procedure the patients were allocated through a scheme provided by the computer programme.

\section{Data collection}

Opioid consumption peroperatively and during a patient's stay at the recovery is registered by the anaesthesiologist in the patients file. The PCA pump is managed by dedicated anaesthesiologic nurse practitioners, who register the opioid consumption from the PCA pump in the patients file. Furthermore, all medication administered at the regular ward, among which are oral opioids, is registered by nurses in the patients file. Pain and DOS scores will be taken by nurses, after which they will be registered in the patients file. All perioperative complications and adverse events will be registered daily in the patients file. Therefore, the primary outcome measure, opioid consumption, will be collected from the patients file. The secondary outcomes, pain and DOS scores, perioperative complications, adverse events and baseline demographic values such as gender, age, weight, details regarding the surgery, the duration of surgery and the length of hospital stay will also be collected from the patients file. All questionnaires and the pain diary will be filled in web-based through the electronic data capture system Castor EDC, after which data will automatically be stored. All data will be recorded using an electronic case report form, web-based, in Castor EDC. Data will be stored coded, which warrants privacy of participants.

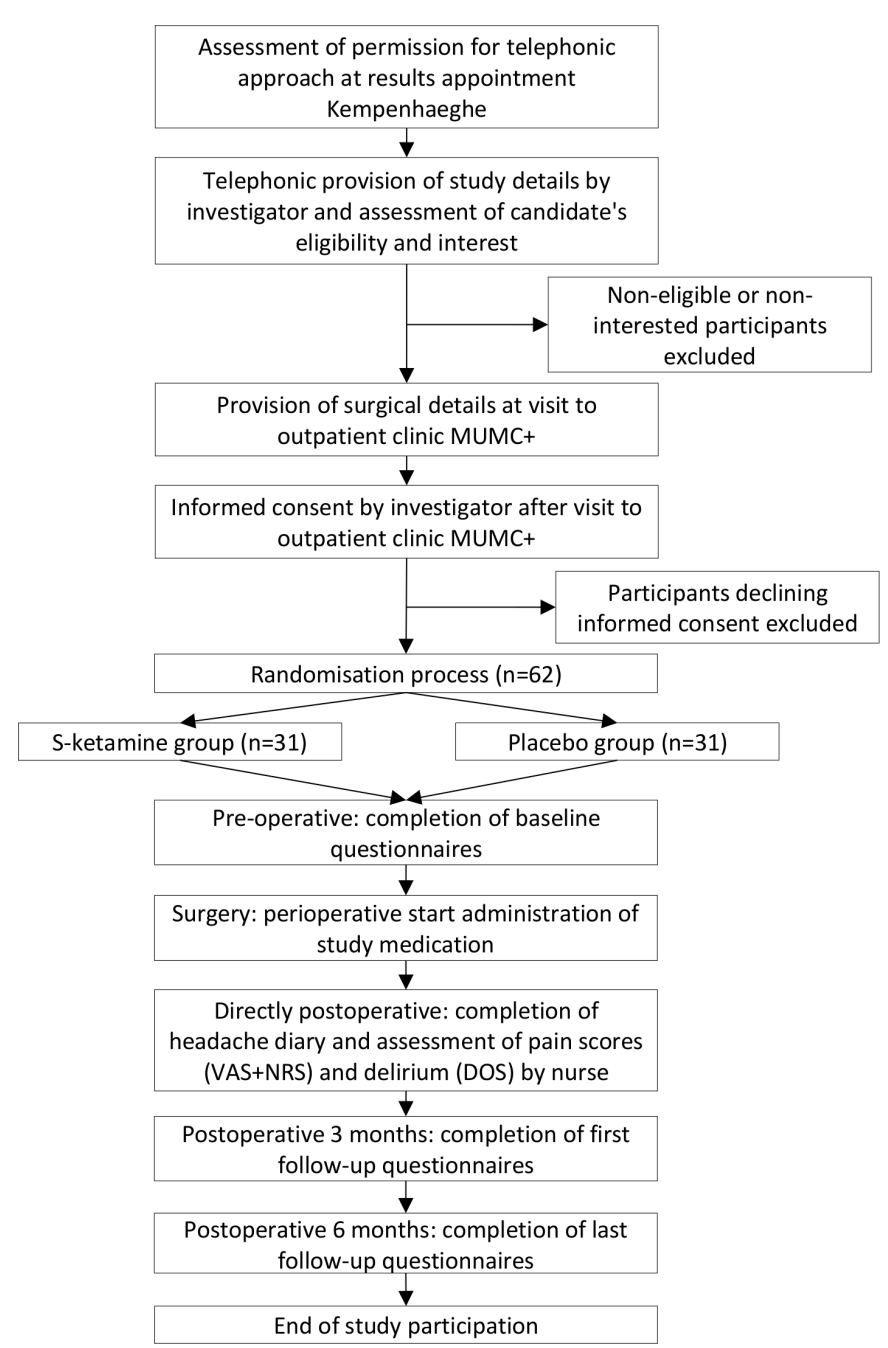

Figure 2 Participant timeline. DOS, delirium observation screening; MUMC+, MaastrichtUniversity Medical Centre+; NRS, Numeric Rating Scale; VAS, Visual AnalogueScale. 


\section{Statistics}

At baseline, normally distributed variables will be presented as mean values and SD. Non-normally distributed variables will be presented as median and range. Categorical data will be presented as frequencies percentages. All collected variables will be presented as a total for the entire study population and stratified by randomised subgroup. There will be no formal testing for statistical differences between the randomised groups.

We will perform all analyses on the intention-to-treat sample. In case study medication has to be terminated and regular s-ketamine protocol will be started, this will have no effect on the intention-to-treat analysis and will be regarded as cointervention. Linear mixed-effects regression will be used to test for statistical differences between the groups, taking the longitudinal nature of the data into account (five measurements). This will be performed based on the primary outcome measure: standards of opioid consumption. In case of clinically meaningful imbalance in baseline characteristics between groups, these variables will be regarded as potential confounders, and will be taken into account using multivariable linear mixed-effects regression. The assumption of a multivariate normally distributed outcome will be checked by visually assessing the histogram of residuals and $\mathrm{Q}-\mathrm{Q}$ plots. In case of clear violation the outcome variable will be transformed using a transformation that solves the non-normality of residuals. Differences are considered to be statistically significant when $p \leq 0.05$. Missing data will not be replaced. Analyses will be performed using SPSS V.23 for Windows. We will consider performing a post hoc subgroup analysis on the difference in opioid consumption between patients who underwent a standard anterior temporal resection or a maximal temporal resection. Results of these analyses will be considered preliminary as the sample size calculation was performed on the main analysis and power is likely to be insufficient.

\section{Interim analysis and preterm termination}

No interim analyses will be performed. Due to the small size of the study sample and the required large difference in primary outcome measure, interim analysis is expected to show no significant difference in the primary outcome measure prior to 3 years termination of the study. S-ketamine is a registered drug administered in daily practice, hence, preterm termination of the study due to unwanted side effects of s-ketamine is not expected.

\section{Monitoring and auditing}

Monitoring will be performed by a member of the local data monitoring committee, from the Clinical Trial Centre Maastricht. Prior to the start of the trial, the study has been classified as low risk. Monitoring will be performed at least three times during the study. Once after the first three patients have been included, once 1.5 year after trial onset and one last time at the end of the inclusion period. Monitoring visits include control of data collection, informed consent forms, compliance to the protocol and applicable laws and regulations and the control of general rights and well-being of trial participants. A monitoring visit report will be sent to the local medical ethical committee and the principal investigator. Unannounced audits can be performed by the audit team of the Clinical Trial Centre Maastricht.

\section{Harms}

Any adverse events reported spontaneously by the subject or observed by the hospital staff will be registered. The most important potential expected adverse event due to s-ketamine is the occurrence of hallucinations. All serious adverse events (SAEs) will be registered and reported through ToetsingOnline to the local medical ethical committee. Life-threatening SAEs or SAEs resulting in death of the patient will be reported within 7 days of first knowledge, followed by a period of maximum 8 days to complete the initial preliminary report. All other SAEs will be reported within a period of maximum 15 days after the sponsor has first knowledge of the SAE.

Liability and subject insurances are provided.

\section{Patient and public involvement}

This research protocol was developed without the involvement of patients or the public. Patients were not invited to contribute to the design of the research methods, material and design or to the writing and editing of any documents.

\section{ETHICS AND DISSEMINATION \\ Research ethics approval and amendments}

The study had been given the following ID: NL61666.068.17. Institutional approval has been given by the MUMC+ Board of Directors on 3 April 2018. A significant modification in the study protocol has been submitted as a substantial amendment to the local medical ethical committee, which has given approval on 6 June 2018. Participants will be included from August 2018 until 2021. Any further significant modifications in the study protocol or significant modifications in other study documents will be submitted for approval to the local medical ethical committee. Subsequently, all study participants will be notified and informed consent will be requested again when necessary. This study will be conducted in accordance with the Dutch Medical Research Involving Human Subjects Act (Wet Medisch-wetenschappelijk Onderzoek met mensen) and the Declaration of Helsinki.

\section{Confidentiality and access to data}

Patient information will be coded and the key to the code that makes traceability possible is saved in a password-locked file and only accessible to the principal investigators. All patient data will be recorded using Castor EDC. Recorded data will be stored using a secured database and safeguarded. Informed consent forms are stored in a locked closet in a locked room (principal investigators room). All research related data and forms will be 
saved for 15 years and nihilated after this period. Research related data and forms are accessible to the investigators, monitors and auditors of the Clinical Trial Centre Maastricht, the Dutch Healthcare authority (Inspectie voor de Gezondheidszorg), and the local medical ethical committee.

\section{Dissemination policy}

The study has been registered with the Dutch National Trial Register (NTR) and been assigned the following ID: NTR6480. Furthermore, the results of this trial will be publicly disclosed and submitted for publication in international peer-reviewed scientific journals.

\section{TRIAL REGISTRATION DATA SET}

Primary registry and trial identifying number: NTR6480. Date of registration in primary registry: 4 July 2017.

Secondary identifying numbers: 2017-002616-13.

Source(s) of monetary or material support: Maastricht Universitair Medisch Centrum+.

Primary sponsor: Maastricht Universitair Medisch Centrum+.

Secondary sponsor: Not applicable.

Contact for public and scientific queries: JS, jiske. sloekers@mumc.nl.

Public title: S-ketamine for acute and chronic headache after brainsurgery.

Scientific title: The effect of perioperative intravenous s-ketamine on acute and chronic postoperative craniotomy pain compared to placebo.

Countries of recruitment: The Netherlands.

Health condition(s) or problem(s) studied: Postcraniotomy pain.

Intervention: Active comparator: s-ketamine.

Placebo comparator: $\mathrm{NaCl} 0.9 \%$.

Key inclusion and exclusion criteria: Ages eligible for study: $\geq 18$ years; Sexes eligible for study: both; Accepts healthy volunteers: no.

Inclusion criteria: Age $>18$ years, elective resective surgery for drug-resistant TLE, drug-resistant epilepsy, based on: (1) chronic, focal epilepsy; (2) not seizure free with antiepileptic medication; (3) no medication options due to adverse effects, signed informed consent for trial participation.

Exclusion criteria: Declined informed consent, allergy to any of the trial medications, current chronic pain, such as, but not limiting to, migraine or other headaches, chronic pain treatment with use of different kinds of pain medication, alcohol, hard or soft drug abuses, inability to complete questionnaires or language barrier, history of psychiatric complaints for which treatment was performed, history of craniotomy or subdural electrode implantation.

Study type: Interventional; allocation: randomised; intervention model: parallel assignment; masking: double blind; primary purpose: treatment; phase IV.

Date of first enrolment: 14-08-2018.

Target sample size: 62 .
Recruitment status: Recruiting.

Primary outcome(s): Total postoperative opioid consumption at the seventh postoperative day with interim measurements at 24, 48, 72 and 96 hours.

Key secondary outcome(s): Postoperative pain intensity scores (VAS+NRS), patient health-related quality of life, psychological parameters, length of hospital stay and adverse events.

\section{Author affiliations}

${ }^{1}$ Neurosurgery, Maastricht Universitair Medisch Centrum+, Maastricht, The Netherlands

${ }^{2}$ Anaesthesiology, Maastricht Universitair Medisch Centrum+, Maastricht, The Netherlands

${ }^{3}$ MHeNS School for Mental Health and Neuroscience, Maastricht University, Maastricht, The Netherlands

${ }^{4}$ Epidemiology, Maastricht University, Maastricht, The Netherlands

${ }^{5}$ Clinical Epidemiology and Medical Technology Assessment, Maastricht Universitair

Medisch Centrum+, Maastricht, The Netherlands

${ }^{6}$ Academic Centre for Epileptology, Maastricht Universitair Medisch Centrum+, Maastricht, The Netherlands

${ }^{7}$ Academic Centre for Epileptology, Kempenhaeghe, Heeze, The Netherlands

${ }^{8}$ Neurology, Maastricht Universitair Medisch Centrum+, Maastricht, The Netherlands

Contributors JCTS: coordinating investigator, concept, drafting and critically revising of protocol. OEMGS: principal investigator, performing surgery, concept, drafting and critically revising of protocol. MB: principal investigator, concept, drafting and critically revising of protocol. GH and SvK: drafting and critically revising of protocol. CB: concept, drafting and critically revising of protocol. $M T, K R$, $A C$ and RPWR: critically revising of protocol. JD: performing surgery and critically revising of protocol.

Funding The authors have not declared a specific grant for this research from any funding agency in the public, commercial or not-for-profit sectors.

Competing interests None declared.

Patient consent for publication Not required.

Ethics approval Ethical approval has been given by the local medical ethical committee academisch ziekenhuis Maastricht/Maastricht University (METC azM/ UM) on 14 March 2018.

Provenance and peer review Not commissioned; externally peer reviewed.

Open access This is an open access article distributed in accordance with the Creative Commons Attribution Non Commercial (CC BY-NC 4.0) license, which permits others to distribute, remix, adapt, build upon this work non-commercially, and license their derivative works on different terms, provided the original work is properly cited, appropriate credit is given, any changes made indicated, and the use is non-commercial. See: http://creativecommons.org/licenses/by-nc/4.0/.

\section{REFERENCES}

1. Dunbar PJ, Visco E, Lam AM. Craniotomy procedures are associated with less analgesic requirements than other surgical procedures. Anesth Analg 1999;88:335-40.

2. de Oliveira Ribeiro MdoC, Sallum AMC, Sallum AMC, et al. Immediate post-craniotomy headache. Cephalalgia 2013;33:897-905.

3. Rocha-Filho PAS, Gherpelli JLD, de Siqueira JTT, et al. Postcraniotomy headache: a proposed revision of IHS diagnostic criteria. Cephalalgia 2010;30:560-6.

4. Gee JR, Ishaq Y, Vijayan N. Postcraniotomy headache. Headache 2003;43:276-8.

5. Molnár L, Simon Éva, Nemes R, et al. Postcraniotomy headache. J Anesth 2014;28:102-11.

6. Headache Classification Committee of the International Headache Society (IHS). The International classification of headache disorders, 3rd edition (beta version). Cephalalgia 2013;33:629-808.

7. Rocha-Filho PAS, Gherpelli JLD, de Siqueira JTT, et al. Postcraniotomy headache: characteristics, behaviour and effect on quality of life in patients operated for treatment of supratentorial intracranial aneurysms. Cephalalgia 2008;28:41-8. 
8. Kaur A, Selwa L, Fromes G, et al. Persistent headache after supratentorial craniotomy. Neurosurgery 2000;47:633-6.

9. Rocha Filho PAS. Post-craniotomy headache after surgery for treatment of cerebral aneurysms. Arq Neuropsiquiatr 2007;65:921-2.

10. Nemergut EC, Durieux ME, Missaghi NB, et al. Pain management after craniotomy. Best Pract Res Clin Anaesthesiol 2007;21:557-73.

11. Umamaheswara Rao GS, Gelb AW. To use or not to use: the dilemma of NSAIDs and craniotomy. Eur J Anaesthesiol 2009;26:625-6.

12. Williams DL, Pemberton E, Leslie K. Effect of intravenous parecoxib on post-craniotomy pain. Br J Anaesth 2011;107:398-403.

13. Jellish WS, Leonetti JP, Kristina S, et al. Morphine/ondansetron PCA for postoperative pain, nausea, and vomiting after skull base surgery. Otolaryngol Head Neck Surg 2006;135:175-81.

14. Laskowski K, Stirling A, McKay WP, et al. A systematic review of intravenous ketamine for postoperative analgesia. Can $J$ Anesth/J Can Anesth 2011;58:911-23.

15. Hayashi $\mathrm{Y}$, Kawaji K, Sun L, et al. Microglial $\mathrm{Ca}(2+)$-activated $\mathrm{K}(+)$ channels are possible molecular targets for the analgesic effects of S-ketamine on neuropathic pain. J Neurosci 2011;31:17370-82.

16. Cohen SP, Bhatia A, Buvanendran A, et al. Consensus guidelines on the use of intravenous ketamine infusions for chronic pain from the American Society of regional anesthesia and pain medicine, the American Academy of pain medicine, and the American Society of Anesthesiologists. Reg Anesth Pain Med 2018;43:521-46.

17. McNicol ED, Schumann R, Haroutounian S. A systematic review and meta-analysis of ketamine for the prevention of persistent postsurgical pain. Acta Anaesthesiol Scand 2014;58:1199-213.

18. Karcioglu M, Davarci I, Tuzcu K, et al. Addition of ketamine to propofol-alfentanil anesthesia may reduce postoperative pain in laparoscopic cholecystectomy. Surg Laparosc Endosc Percutan Tech 2013:23:197-202.10.1097/SLE.0b013e3182827f09
19. Jouguelet-Lacoste J, La Colla L, Schilling D, et al. The use of intravenous infusion or single dose of low-dose ketamine for postoperative analgesia: a review of the current literature. Pain Med 2015;16:383-403.

20. Ferreira-Valente MA, Pais-Ribeiro JL, Jensen MP. Validity of four pain intensity rating scales. Pain 2011;152:2399-404.

21. van Seventer R, Vos C, Giezeman M, et al. Validation of the Dutch version of the DN4 diagnostic questionnaire for neuropathic pain. Pain Pract 2013;13:390-8.

22. Bouhassira D, Attal N, Alchaar $\mathrm{H}$, et al. Comparison of pain syndromes associated with nervous or somatic lesions and development of a new neuropathic pain diagnostic questionnaire (DN4). Pain 2005;114:29-36.

23. Radloff LS. The CES-D scale: a self-report depression scale for research in the general population. Applied psychological measurement 1977;1:385-401.

24. Theunissen M, Peters ML, Schouten EGW, et al. Correction: validation of the surgical fear questionnaire in adult patients waiting for elective surgery. PLoS One 2016;11:e0162737.

25. Sullivan MJL, Bishop SR, Pivik J. The pain catastrophizing scale: development and validation. Psychol Assess 1995;7:524-32.

26. VanderZee KI, Sanderman R, Heyink JW, et al. Psychometric qualities of the Rand 36-Item health survey 1.0: a multidimensional measure of general health status. Int J Behav Med 1996;3:104.

27. Cleeland CS, Ryan KM. Pain assessment: global use of the brief pain inventory. Ann Acad Med Singapore 1994;23:129-38.

28. Schuurmans MJ, Shortridge-Baggett LM, Duursma SA. The delirium observation screening scale: a screening instrument for delirium. Res Theory Nurs Pract 2003;17:31-50. 